TO ANALYSE PATIENT OF POST CHOLECYSTECTOMY SYMPTOMS AFTER CHOLECYSTECTOMY

\section{General Surgery}

Dr. Jitendra K. Mangtani

Dr Ketan Patel*
Professor, Head of Unit, Department of General Surgery, MGMCH, Jaipur, Rajasthan, (India)

JR $3^{\text {rd }}$ Year, Department of General Surgery, MGMCH, Jaipur, Rajasthan, (India) *Corresponding Author

Dr. Anshul Mathur JR $3^{\text {rd } Y e a r, ~ D e p a r t m e n t ~ o f ~ G e n e r a l ~ S u r g e r y, ~ M G M C H, ~ J a i p u r, ~ R a j a s t h a n, ~(I n d i a) ~}$

Dr.Heet Amlani $\quad$ JR $2^{\text {rd } Y e a r, ~ D e p a r t m e n t ~ o f ~ G e n e r a l ~ S u r g e r y, ~ M G M C H, ~ J a i p u r, ~ R a j a s t h a n, ~(I n d i a) ~}$

Dr. Vartika Gupta $5 J R 1^{\text {rd } Y e a r, ~ D e p a r t m e n t ~ o f ~ G e n e r a l ~ S u r g e r y, ~ M G M C H, ~ J a i p u r, ~ R a j a s t h a n, ~(I n d i a) ~}$

Dr. Salman Uddin $\quad 6 \mathrm{JR} 1{ }^{\text {rd }}$ Year, Department of General Surgery, MGMCH, Jaipur, Rajasthan, (India)

Introduction: Post-cholecystectomy syndrome (PCS) comprise of a group of abdominal symptoms that occur after cholecystectomy. Post cholecystectomy syndrome is defined as group of heterogeneous symptoms. It is consists of upper abdominal pain, vomiting and dyspepsia, which occur after cholecystectomy. Although, this term is not accurate, as it comprises biliary and non-biliary disorders, possibly not related to cholecystectomy. AIM : To analyse the clinical evaluation for post cholecystectomy Symptoms. Objectives : To identify the causes for post cholecystectomy symptoms.To identify pre-operative factors (h/o ERCP, attacks of cholecystitis) associated with post cholecystectomy symptoms. Materials \& Methods : A Prospective clinical hospital based study was done in 100 patients at tertiary care referral hospital in the department of general surgery. All cases of post- cholecystectomy who had clinical symptoms of PCS Cases in which on follow up USG and LFT were done. Result: In our study 100 patients were taken who developed PCS after cholecystectomy. Among all patients, $16 \%$ patient developed PCS due to biliary etiology. Among $16 \%$ patients most common cause is recurrent CBD stone (6\%) and second most common causes is retained CBD Stone (3\%) \& spincter Of Oddi dysfunction (3\%). Discussion : This analysis provides a qualitative overview of etiologies of abdominal symptoms after cholecystectomy. Based on the etiologies of persistent and incident symptoms after cholecystectomy provided in this review, we identified the cause of long-term symptoms after cholecystectomy. Conclusion : In our study, PCS developed mainly due to non-biliary etiology considered $84 \%$ and biliary etiology considered only $16 \%$. Female has higher risk to developing post cholecystectomy symptoms compared to male after cholecystectomy.

\title{
KEYWORDS
}

Cholecystectomy, post cholecystectomy syndrome, CBD stone

\section{Introduction}

Post-cholecystectomy syndrome (PCS) comprise of a group of abdominal symptoms that occur after cholecystectomy. Post cholecystectomy syndrome is defined as group of heterogeneous symptoms. It is consists of upper abdominal pain, vomiting and dyspepsia, which occur after cholecystectomy. Although, this term is not accurate, as it comprises biliary and non-biliary disorders, possibly not related to cholecystectomy.

The incidence of post cholecystectomy symptoms has been reported to be as high as $40 \%$ in one study, and the onset of symptoms may range from 2 days to 25 years. There may also be gender-specific risk factors for developing symptoms after cholecystectomy. The clinical management of post cholecystectomy syndrome patients is frequently with no evidence-based approach.

Biliary manifestations of PCS usually occur because of operative complications in early post-operative period, such as bile duct injury / biliary leakage. A late biliary manifestations is commonly caused by inflammatory strictures involving the sphincter of Oddi and the common bile duct, recurrent stone or biliary dyskinesia.

The most common non biliary cause of PCS is peptic ulcer and the most common biliary cause is ductal calculi. Symptomatic treatment is required for patient who presented with PCS and a complete workup is required to diagnose the actual cause of symptoms; LFT and abdominal USG are the early diagnostic tests that are ordered, and further investigations accordingly.

\section{Aim}

- To analyse the clinical evaluation for post cholecystectomy Symptoms.

\section{Objectives}

- To identify the causes for post cholecystectomy symptoms.

- To identify pre-operative factors (h/o ERCP, attacks of cholecystitis) associated with post cholecystectomy symptoms.

\section{Materials \& Methods}

A Prospective clinical hospital based study was done in 100 patients at tertiary care referral hospital in the department of general surgery.

Study design: Prospective study

Study period: January 2019 till June 2020

\section{Inclusion Criteria:}

1. All cases of post-cholecystectomy who had clinical symptoms of PCS.

2. Cases in which on follow up USG and LFT were done.

\section{Exclusion criteria:}

1. Patients in whom LFT and USG were not done.

2. Patients who do not give consent for study.

This study conducted on 100 patients who developed PCS after cholecystectomy. Patient were chosen randomly irrespective of their age and sex.

All patients have been evaluated with a detailed history of their illness, onset and duration of presenting symptoms. USG was done in all patients to confirm the diagnosis. All of them were planned to undergo cholecystectomy. Follow up of patients done by outpatient basis. Persistent symptoms diagnosed by USG abdomen, LFT and ERCP.

Appropriate statistical tests will be applied to find significant association. P value $<0.05$ will be considered statistically significant.

\section{Result}

In our study 100 patients were taken who developed PCS after cholecystectomy. Among all patients, $16 \%$ patient developed PCS due to biliary etiology. Among $16 \%$ patients most common cause is recurrent $\mathrm{CBD}$ stone $(6 \%)$ and second most common causes is retained CBD Stone (3\%) \& spincter of Oddi dysfunction (3\%).(Table-1)

Table 1 : Biliary Etiology 


\begin{tabular}{|c|c|c|}
\hline & Number of cases & Percentage \\
\hline $\begin{array}{c}\text { RETAINED CBD } \\
\text { STONE }\end{array}$ & 3 & 3.00 \\
\hline $\begin{array}{c}\text { RECURRENT CBD } \\
\text { STONE }\end{array}$ & 6 & 6.00 \\
\hline CBD STRICTURE & 2 & 2.00 \\
\hline BILE LEAKAGE & 2 & 2.00 \\
\hline $\begin{array}{c}\text { SPINCTER OF ODDI } \\
\text { DYSFUNCTION }\end{array}$ & 3 & 3.00 \\
\hline TOTAL & 100 & 100.00 \\
\hline
\end{tabular}

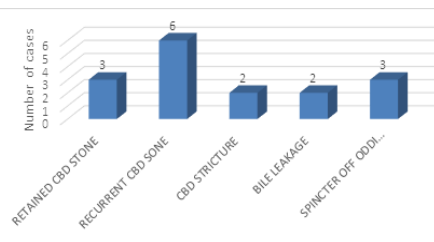

$84 \%$ patients developed PCS due to non biliary etiology, among $84 \%$ patients most common cause is gastritis $(42 \%)$ which shows that most of the postcholecystectomy symptoms developed from non- biliary etiology.

Table 2 : Non Biliary Etiology

\begin{tabular}{|c|c|c|}
\hline & Number of cases & Percentage \\
\hline GASTRITIS & 42 & 42.00 \\
\hline PANCREATITIS & 2 & 2.00 \\
\hline PEPTIC ULCER & 18 & 18.00 \\
\hline $\begin{array}{c}\text { NO OBVIOUS } \\
\text { CAUSE }\end{array}$ & 22 & 22.00 \\
\hline TOTAL & 100 & 100.00 \\
\hline
\end{tabular}

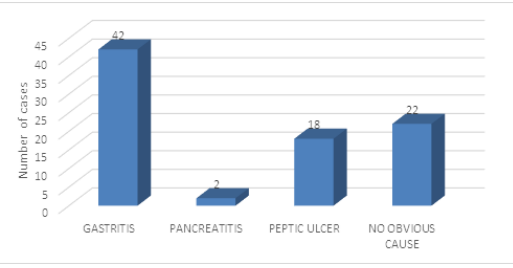

Among all patients, 42 were diagnosed by gastritis, 18 patients were diagnosed by peptic ulcer and 2 patients were diagnosed by pancreatitis and they were managed conservatively. (Table-2)

\section{Table 5 : Risk Factor}

\begin{tabular}{|c|c|c|}
\hline & Number of Cases & Percentage \\
\hline H/O ERCP & 23 & 23.00 \\
\hline OBESITY & 15 & 15.00 \\
\hline DELAY OPERATION & 5 & 5.00 \\
\hline $\begin{array}{c}\text { ATTACK OF } \\
\text { CHOLECYSTITIS }\end{array}$ & 16 & 16.00 \\
\hline $\begin{array}{c}\text { NO OBVIOUSRISK } \\
\text { FACTOR }\end{array}$ & 41 & 41.00 \\
\hline Total & 100 & 100.00 \\
\hline
\end{tabular}

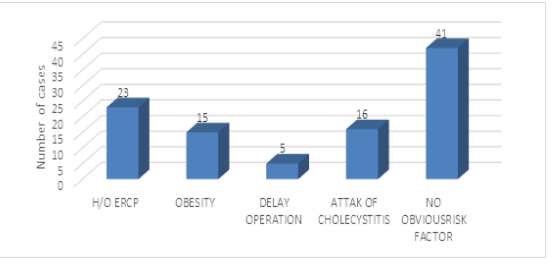

- Among 100 patients, 41 patients are those who don't have any obvious risk factor, 23 patients have previous h/o ERCP, 16 Patients have previous attack of cholecystitis. 15 patient have obesity who developed PCS.(Table-3)

\section{Discussion}

This analysis provides a qualitative overview of etiologies of abdominal symptoms after cholecystectomy. Based on the etiologies of persistent and incident symptoms after cholecystectomy provided in

this review, we identified the cause of long-term symptoms after cholecystectomy.

In our study, we categorized etiology of long-term postoperative symptoms after cholecystectomy. We established that most persisting symptoms are likely to be caused by non biliary etiology as compared to biliary etiology. Detailed anamnesis and tailored diagnostic tests (such as ultrasound, ERCP, LFT, pancreatic marker and upper GI endoscopy) will provide insight in the presence of abdominal disorders.

Analysis of our cohort during follow-up showed that there were two constant factors (previous attacks of acute cholecystitis and previous $\mathrm{h} / \mathrm{o}$ ERCP) that significantly affected the development of PCS. Prior attacks of acute cholecystitis have been associated with PCS, and the mechanism by the theory of referred pain from the gallbladder which continuing or manifesting post-operatively.

In study conducted by S.S. Jaunoo, Bile duct injury which are seen $0.4 \%$ to $4 \%$ and retained/recurrent CBD stone seen $1.2 \%$ to $14 \%$.

In the our study, we had taken all follow up 100 patients of abdominal pain after cholecystectomy. Among all patients 6 patients were diagnosed by recurrent $\mathrm{CBD}$ stone, 3 patients were diagnosed by retained $\mathrm{CBD}$ stone, 2 were diagnosed by bile leakage, 2 were diagnosed by CBD stricture.

In study conducted by S.S. Jaunoo, the most common cause of PCS is extrabiliary disorders such as gastritis, peptic ulcer, irritable bowel syndrome or pancreatitis ranging from $1 \%$ to $75 \%$.

In our study, we had taken all follow up 100 patients of abdominal pain after cholecystectomy and they were evaluated by LFT, pancreatic enzyme, USG, ERCP and upper GI endoscopy. Among all patients 42 were diagnosed by gastritis, 18 patients were diagnosed by peptic ulcer and 2 patients were diagnosed by pancreatitis and they were managed conservatively. Those patients who had PCS due to biliary etiology were managed by ERCP.

\section{Conclusion}

In our study, PCS developed mainly due to non-biliary etiology considered $84 \%$, and biliary etiology considered only $16 \%$. Female has higher risk to developing post cholecystectomy symptoms compared to male after cholecystectomy. Among all causes, gastritis is most common cause of developing PCS. Patients who were at higher risk of developing PCS, had previous h/o ERCP, attacks of cholecystitis and Obesity. Half of the patients develops PCS has no identified previous risk factor.

\section{References}

1. Schofer JM. Biliary causes of postcholecystectomy syndrome. J Emerg Med. 2008 Aug 22

2. Terhaar OA, Abbas S, Thornton FJ, Duke D, O'Kelly P, Abdullah K. Imaging patients with "post-cholecystectomy syndrome": an algorithmic approach. Clin Radiol 2005;60:78-84

3. T. Yamada (Ed.), Textbookgastroenterology (2nded.), Lippincott, Ph iladelphi(1995)

4. P.H. Zhou, F.L. Liu, L.Q. Yao, X.Y. QinEndoscopic diagnosis and treatment of postcholecystectomy syndrome Hepatobiliary Pancreat Dis Int, 2 (2003), pp. 117-120 R Girometti, MD, G Brondani, MD, L Cereser, MD, G Como, MD, M Del Pin,

MD, M Bazzocchi, MD, and C Zuiani, MDBr J Radiol. Post- cholecystectomy syndrome: spectrum of biliary findings at magnetic resonance syndrome: spectrum of biliary findings

6. Divya Arora, Robin Kaushik, Ravinder Kaur, Atul Sachdev Post- cholecystectomy syndrome: Anew look at an old problem Access Surg 2018;14:202-7

7. S.S.JaunooS.MohandasL.M.AlmondPostcholecystectomy syndrome (PCS) International Journal of Surgery, Volume 8, Issue 1, 2010.

8. Murshid K R.the post cholecystectomy syndrome:a review of $\mathrm{S}$ gastroenterology1996;2:124-37 